\title{
Influence of autoionisation and predissociation on the photoelectron parameters in $\mathbf{H B r}$
}

\author{
H Lefebvre-Brion†, M Salzmann $\ddagger$, H-W Klausing $\ddagger$, M Müller \\ $\mathrm{N}$ Böwering $\ddagger$ and $U$ Heinzmann $\ddagger$ \\ † Laboratoire de Photophysique Moléculaire, Bâtiment 213, Université de Paris-Sud, 91405 \\ Orsay, France \\ $\doteqdot$ Universität Bielefeld, Fakultät für Physik, D-4800 Bielefeld and Fritz-Haber-Institut der \\ MPG, D-1000 Berlin 33, Federal Republic of Germany
}

Received 5 July 1989

\begin{abstract}
The photoionisation spectrum of $\mathrm{HBr}$ is strongly influenced in the $100-85 \mathrm{~nm}$ wavelength range by the autoionisation of Rydberg series converging to the $A^{2} \Sigma^{+}$ionic state. These Rydberg series are also partly predissociated. Using a unified multichannel quantum-defect theory treatment of autoionisation and predissociation processes, both the photoionisation and photodissociation cross sections are calculated. The photodissociation cross section is predicted to be 10 times larger than for $\mathrm{HCl}$, i.e. about $10 \mathrm{Mb}$. The photoelectron angular distribution parameter $\beta$ is predicted and the influence of predissociation on its value is pointed out. The spin-polarisation parameter $A$ (or $\bar{P}$ ) is calculated. It shows oscillatory structure related to the autoionisation of the ${ }^{1} \Pi$ states. Using circularly polarised synchrotron radiation, this parameter has been measured for both fine-structure states $\mathrm{HBr}^{+} \mathrm{X}^{2} \Pi_{3 / 2}(v=0)$ and $\mathrm{X}^{2} \Pi_{1 / 2}(v=0)$. The agreement between calculated and measured values is relatively good.
\end{abstract}

\section{Introduction}

The photoionisation spectrum of $\mathrm{HBr}$ presents particular interest because both theoretical calculations and experiments can be readily performed for this molecule. Another reason for interest is that the ground state $X^{2} \Pi$ of the ion has a large spin-orbit splitting $\left(2651 \mathrm{~cm}^{-1}\right)$. This facilitates measurements on the spin-polarisation parameters of the photoelectron, which require separation of the fine-structure components. The energy region between the ${ }^{2} \Pi_{3 / 2}$ and ${ }^{2} \Pi_{1 / 2}$ ionisation thresholds was previously investigated and good agreement between theory and experiment was obtained for the spin-orbit autoionisation structure (Lefebvre-Brion et al 1986).

In this paper, we investigate the wavelength region above the ${ }^{2} \Pi$ thresholds between 100 and $85 \mathrm{~nm}$, which is dominated by autoionisation resonances belonging to Rydberg series converging to the $\mathrm{A}^{2} \Sigma^{+}$excited state of $\mathrm{HBr}^{+}$. In $\mathrm{HCl}$, the corresponding autoionisation features were studied previously and comparison between theory and experiment yielded an assignment for these peaks (Lefebvre-Brion et al 1988). More recently, the photodissociation cross section for $\mathrm{HCl}$ was measured in this same energy region (White et al 1987, Frohlich et al 1989). A theoretical treatment of both autoionisation and predissociation processes was made and it was shown that the $\Pi$ states are autoionised and the ${ }^{1} \Sigma^{+}$states are preferentially predissociated (LefebvreBrion and Keller 1989). 
In molecular photoionisation, measurements of the photoelectron spin-polarisation parameter $A$ (also denoted $\bar{P}$ ) were carried out previously for $\mathrm{CO}_{2}, \mathrm{~N}_{2} \mathrm{O}$ and $\mathrm{CH}_{3} \mathrm{Br}$ (Heinzmann et al 1980, 1981) for the spin-orbit autoionisation region, and for $\mathrm{HI}$ in the range above the ${ }^{2} \Pi$ thresholds (Böwering et al 1988). The spin-polarisation data for HI show a strong variation due to electronic autoionisation (Böwering et al 1989). A general formalism within the non-relativistic theory for the spin polarisation of the photoelectrons from diatomic molecules was derived by Cherepkov (1981) and calculations have been reported for $\mathrm{HI}$ and $\mathrm{HBr}$ (Raseev et al 1987) without including the electronic autoionisation.

For $\mathrm{HBr}$, the absorption spectrum was analysed by Terwilliger and Smith (1974, 1975 ) and the photoionisation spectrum was reported briefly (Dehmer and Chupka 1978). We present here both a theoretical study of the $\mathrm{HBr}$ photoionisation spectrum and constant-ionic-state (cIs) photoelectron measurements. In $\$ 2$ the experimental apparatus is described. In $\S 3$ the background of the theory is reviewed and finally in $\S 4$ the experimental and theoretical results for the photoionisation cross section and for the spin-polarisation parameter $A$ (or $\bar{P}$ ) of the photoelectron are compared. Predictions are made for the photodissociation cross section and for the angular asymmetry parameter $\beta$.

\section{Experimental details}

The photoelectron spin-polarisation parameter $A$ (or $\bar{P}$ ) was measured in the wavelength region of $100-85 \mathrm{~nm}$ by performing angle- and spin-resolved photoelectron spectroscopy. The experiment was carried out with circularly polarised synchrotron radiation emitted out of the plane of the storage ring BESSY with an apparatus described in detail previously (Heckenkamp et al 1986). Briefly, an effusive molecular beam of $\mathrm{HBr}(99.8 \%$ purity) is crossed by vacuum-ultraviolet radiation at the exit slit of a $6.5 \mathrm{~m}$ normal-incidence monochromator (Schäfers et al 1986). A liquid-nitrogen cold trap is used to freeze the gas after crossing of the excitation region; the background pressure inside the vacuum chamber is less than $6 \times 10^{-5}$ mbar. The photoelectrons emitted at reaction angle $\theta$ are energy-analysed by a rotatable hemispherical spectrometer (Jost 1979) with an energy resolution of $\Delta E \simeq 100 \mathrm{meV}$. For subsequent spin analysis, the electrons are accelerated to $100 \mathrm{keV}$ and the transverse electron spin polarisation is measured in a Mott detector. (Two gold foils with different thicknesses and values of the Sherman function of -0.21 and -0.23 , respectively, were used.) The spin-polarisation parameter $A$ (characterising the component $A(\theta)$ of the spin-polarisation vector parallel to the photon polarisation) can be obtained directly from measurements at the magic angle, $\theta_{\mathrm{m}}=54.7^{\circ}$, using left- or right-handed circularly polarised light and taking into account the degree of circular polarisation of $\left|P_{\text {circ }}\right|=(92 \pm 3) \%$.

Photoelectron spectra taken in the wavelength region examined here showed the spin-orbit split final ionic states $\operatorname{HBr}^{+} \mathrm{X}^{2} \Pi_{3 / 2}(v=0)$ and $\mathrm{X}^{2} \Pi_{1 / 2}(v=0)$ as well as several vibrationally excited peaks, which were about 10 times less intense. Since the value of the spin-orbit splitting is close to the vibrational spacing for these states (Huber and Herzberg 1979) however, peaks belonging to ${ }^{2} \Pi_{1 / 2}(v)$ and ${ }^{3} \Pi_{3 / 2}(v+1)$ could not be resolved. Therefore, the measurements were restricted to photoelectrons corresponding to final ionic states with $v=0$. Separate cis measurements of the relative partial photoionisation cross section for both spin-orbit states indicated nearly identical autoionisation peaks and an almost constant branching ratio. The structures observed 
were in close agreement with the total photoionisation spectrum of Dehmer and Chupka (1978).

The data obtained for the wavelength dependence of the spin-polarisation parameter $A$ were taken in first order of the monochromater $(\Delta \lambda=0.4 \mathrm{~nm})$ for the final ionic states $\mathrm{HBr}^{+} \mathrm{X}^{2} \Pi_{3 / 2}(v=0)$ and $\mathrm{X}^{2} \Pi_{1 / 2}(v=0)$ (denoted $A(1 / 2)$ and $A(3 / 2)$ respectively) and are shown in figure 1 . The data were accumulated at the peak and valley positions of the autoionisation structures of the partial cross sections. As seen in figure 1 , a strong variation in both $A(1 / 2)$ and $A(3 / 2)$ due to autoionisation is observed. In particular, a decrease to small absolute values of the polarisation occurs in the region of $90-95 \mathrm{~nm}$. The results might be affected by instrumental resolution. However, two data points for $A(3 / 2)$ at 91.2 and $90.75 \mathrm{~nm}$ taken with higher resolution in second order of the monochromator were found to be in agreement with the results obtained in first order. This shows that the influence of the limited resolution is not too severe. In general, the data obtained at the same photon energy were found to be in accordance with the non-relativistic relationship (Cherepkov 1981), valid in pure Hund's case (a):

$$
A(1 / 2)=-A(3 / 2)
$$

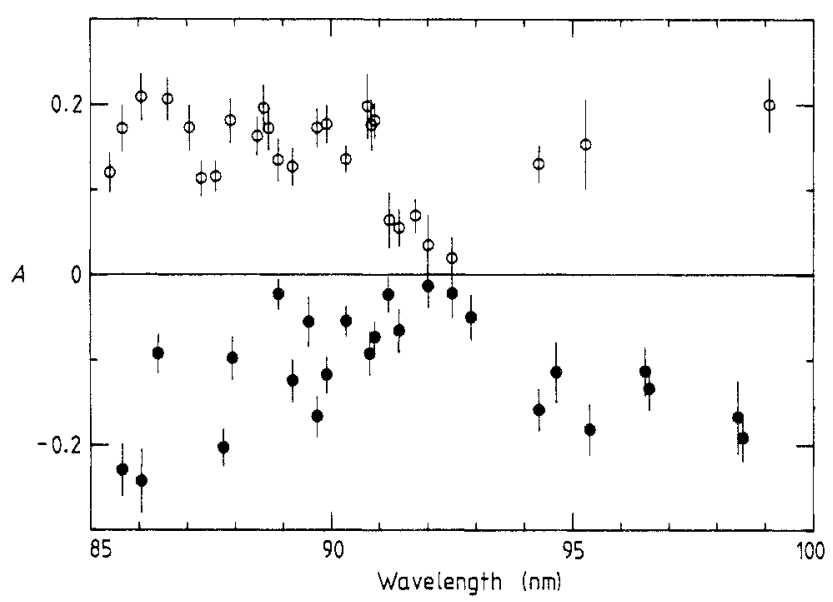

Figure 1. Spin-polarisation parameter $A$ for $\mathrm{HBr}^{+}{ }^{2} \Pi_{3 / 2}(v=0)$ (open circles) and ${ }^{2} \Pi_{1 / 2}$ $(v=0)$ (full circles) final ionic states. The error bars denote the single statistical error including the uncertainty of the Sherman function of the Mott detector and of the light polarisation.

\section{Theoretical framework}

As described in detail previously (Lefebvre-Brion and Keller 1989), the so-called two-step unified multichannel quantum-defect theory (MQDT) (Giusti 1980, GiustiSuzor and Jungen 1984), which has been applied to treat simultaneously the autoionisation and predissociation processes in the $\mathrm{HCl}$ molecule, is used here for the $\mathrm{HBr}$ molecule. The ab initio quantities necessary for a MQDT treatment, namely the quantum defects $\mu_{\beta \lambda}$, the transition moments $D_{\beta \lambda}$ and the $l$-mixing coefficients $U_{1 \lambda, \beta \lambda}$ for each $\beta \lambda$ channel, have already been reported for the channels with the ${ }^{2} \Pi$ ionic core of 
$\mathrm{HBr}^{+}$(Lefebvre-Brion et al 1986) for an electron energy equal to $0.005 \mathrm{au}$. The energy region where the autoionised peaks appear corresponds to a slightly larger electron energy but the photoionisation cross section presents a maximum here (Raseev et al 1987, figure 3) and we have kept these parameters independent of the electron energy. The corresponding parameters for the $\mathrm{A}^{2} \Sigma^{+}$ionic core, and the residual electrostatic interaction $V_{\beta \lambda, \beta^{\prime} \lambda}^{\prime}$, between channels having the same ${ }^{1} \Lambda$ symmetry (here ${ }^{1} \Pi$ and ${ }^{1} \Sigma^{+}$) but belonging to different ionic cores (namely $\mathrm{X}^{2} \Pi$ and $\mathrm{A}^{2} \Sigma^{+}$), are given in table 1 . For simplicity, the $\varepsilon \mathrm{f} \sigma$ and $\varepsilon \mathrm{f} \pi$ channels have not been introduced in the final results, owing to their weak contributions. The vibrational overlaps necessary to weight the electronic transition moments or the electrostatic interactions are given in table 2.

The predissociation process has been assumed to be due, as in $\mathrm{HCl}$, to the Rydberg states converging to the a ${ }^{4} \Pi$ repulsive ionic state. The potential curves of these states have been taken parallel to the calculated curve of a ${ }^{4} I I$ (Banichevich et al 1989). Figure 2 gives the potential curves of this problem. The ${ }^{3} \Pi_{0}$ with configuration $\left({ }^{4} \Pi\right)$ $5 \mathrm{~s} \sigma$ has been assumed to go to the dissociation limit $\mathrm{H}+\mathrm{Br}^{*}\left({ }^{2} \mathrm{P}_{1 / 2}, 5 \mathrm{~s}\right)$, which occurs at $100.7 \mathrm{~nm}$. Also the $\left({ }^{4} \Pi\right) 5 \mathrm{p} \sigma$ state goes to the limit $\mathrm{H}+\mathrm{Br}^{*}\left({ }^{4} \mathrm{P}_{1 / 2}, 5 \mathrm{p}\right)$, which appears at $94.23 \mathrm{~nm}$. These ${ }^{3} \Pi_{0}$ states can dissociate only the ${ }^{1} \Sigma^{+}$states with an $\mathrm{A}^{2} \Sigma^{+}$core. We have assumed that the ${ }^{3} \Sigma_{1}^{+}$state with configuration $\left({ }^{4} \Pi\right) 5 \mathrm{p} \pi$ goes to the same

Table 1. Ab initio calculated parameters for electronic autoionisation for the $\mathrm{A}^{2} \Sigma^{+}$ion core $(R=2.672 \mathrm{au}, \varepsilon=0.0015 \mathrm{au})$.

(a) ${ }^{1}$ II states

\begin{tabular}{lrrr}
\hline$\beta \lambda$ & $\varepsilon \mathrm{p} \pi$ & $\varepsilon \mathrm{d} \pi$ & \multicolumn{1}{c}{$\varepsilon \mathrm{f} \pi$} \\
\hline$\mu_{\beta \lambda}^{0}$ & -0.4023 & 0.3191 & 0.0350 \\
& $(0.651)^{\mathrm{a}}$ & $(0.252)^{\mathrm{a}}$ & \\
$D_{\beta \lambda}^{0}(\mathrm{au})$ & 0.9721 & 3.3597 & 0.1760 \\
$U^{0}{ }^{0}, \beta \lambda$ & 0.9240 & 0.3804 & 0.0355 \\
& -0.3815 & 0.9115 & 0.1483 \\
& 0.0241 & -0.1560 & 0.9354 \\
$V_{\beta^{\prime} \lambda^{\prime}}^{\beta \mathrm{s} \sigma}$ & & & \\
$\varepsilon \mathrm{p} \sigma$ & -0.0290 & -0.0210 & 0.0032 \\
$\varepsilon \mathrm{d} \sigma$ & -0.0090 & -0.0581 & -0.0004 \\
$\varepsilon \mathrm{f} \sigma$ & 0.0244 & 0.0396 & 0.0102 \\
$\varepsilon \mathrm{d} \delta$ & -0.0087 & -0.0062 & 0.0046 \\
\hline
\end{tabular}

(b) ${ }^{1} \Sigma^{+}$states

\begin{tabular}{lrrrr}
\hline$\beta \lambda$ & $\varepsilon \mathrm{s} \sigma$ & $\varepsilon \mathrm{p} \sigma$ & \multicolumn{1}{c}{$\varepsilon \mathrm{d} \sigma$} & \multicolumn{1}{c}{$\varepsilon \mathrm{f} \sigma$} \\
\hline$\mu_{\beta \lambda}^{0}$ & 0.0176 & 0.4928 & -0.3088 & 0.0461 \\
$D_{\beta \lambda}^{0}(\mathrm{au})$ & -0.3323 & 0.8194 & 0.0369 & -0.0734 \\
$U_{i \lambda, \beta \lambda}^{0}$ & 0.7935 & 0.0540 & 0.5075 & 0.1409 \\
& -0.1351 & 0.9740 & 0.1605 & -0.0599 \\
& 0.4524 & 0.2163 & -0.8464 & 0.0522 \\
$V_{\beta^{\prime} \lambda^{\prime}}^{\beta \lambda}$ & 0.2137 & -0.0412 & -0.0168 & -0.9622 \\
$\varepsilon \mathrm{p} \pi$ & & & & \\
$\varepsilon \mathrm{d} \pi$ & 0.0334 & 0.0361 & -0.0299 & 0.0169 \\
$\varepsilon \mathrm{f} \pi$ & -0.0072 & 0.0391 & -0.0619 & -0.0275 \\
\hline
\end{tabular}

${ }^{a}$ Values in parentheses are the values used in the calculations. 
Table 2. Vibrational quantities for the $\mathrm{A}^{2} \Sigma^{+}$state of $\mathrm{HBr}^{+} t$.

\begin{tabular}{llll}
\hline$v^{\prime}$ & $\Delta G\left(\mathrm{~cm}^{-1}\right)$ & $\left\langle v^{\prime} \mid \mathrm{X}^{1} \Sigma^{+}\left(v^{\prime \prime}=0\right)\right\rangle$ & $\left\langle v^{\prime} \mid \mathrm{X}^{2} \Pi\left(v^{\prime \prime}=0\right)\right\rangle$ \\
\hline 0 & 1328.0 & 0.3412 & 0.4549 \\
1 & 1253.0 & 0.4552 & 0.5271 \\
2 & 1177.0 & 0.4630 & 0.4716 \\
3 & 1102.0 & 0.4131 & 0.3740 \\
4 & 1026.0 & 0.3419 & 0.2774 \\
5 & 951.0 & 0.2702 & 0.1980 \\
6 & 875.0 & 0.2078 & 0.1383 \\
7 & & 0.1573 & 0.0956 \\
\hline
\end{tabular}

"Parameters for Morse potentials (from Huber and Herzberg 1979): $\mathrm{HBr} \mathrm{X}^{1} \Sigma^{+}$

$\mathrm{HBr}^{+} \mathrm{X}^{2} \Pi$

$$
\omega_{\mathrm{e}}=2648.975 \mathrm{~cm}^{-1} \quad \omega_{\mathrm{e}} x_{\mathrm{e}}=45.217 \mathrm{~cm}^{-1} \quad r_{\mathrm{e}}=1.41443 \AA
$$

$$
\begin{aligned}
& \omega_{\mathrm{e}}=2441.52 \mathrm{~cm}^{-1} \quad \omega_{\mathrm{e}} x_{\mathrm{e}}=47.4 \mathrm{~cm}^{-1} \quad r_{\mathrm{e}}=1.4484 \AA \\
& 1 \mathrm{P}=94130 \mathrm{~cm}^{-1} \text { for } \mathrm{X}^{2} \Pi_{3 / 2} \quad I P=96781 \mathrm{~cm}^{-1} \text { for } \mathrm{X}^{2} \Pi_{1 / 2}
\end{aligned}
$$
$\mathrm{A}^{2} \mathrm{~s}^{+}$

$$
\begin{aligned}
& \omega_{\mathrm{e}}=1404 \mathrm{~cm}^{-1} \\
& 1 \mathrm{P}=29230 \mathrm{~cm}^{-1}
\end{aligned} \quad \omega_{\mathrm{e}} x_{\mathrm{e}}=37.75 \mathrm{~cm}^{-1} \quad r_{\mathrm{e}}=1.6842 \AA
$$

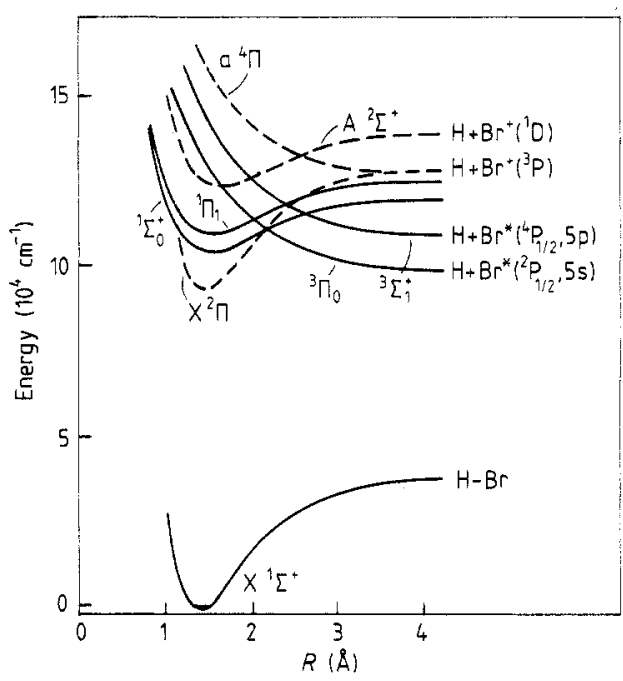

Figure 2. Experimental potential curves of the bound states of $\mathrm{HBr}$ and $\mathrm{HBr}^{+}$. The ${ }^{4} \Pi$ repulsive curve of $\mathrm{HBr}^{+}$is taken from calculations of Banichevich et al (1989). The curves for Rydberg states are taken parallel to the curve of their ionic core.

limit as the last ${ }^{3} \Pi_{0}$ state. It can predissociate the ${ }^{1} \Pi$ states. For simplicity, we have not considered the limit $\mathrm{H}+\mathrm{Br}^{*}\left({ }^{4} \mathrm{D}_{1 / 2}, 5 \mathrm{p}\right)$ at $92.27 \mathrm{~nm}$, which can contribute very much to the dissociation of the $n \mathrm{~d} \pi^{1} \Pi$ states, and the effect of the lowest ${ }^{3} \Pi_{0}$ has been taken into account only up to the second dissociation limit, namely $94.23 \mathrm{~nm}$. The highest dissociation limit included in this study is reported on figure 3 . The spin-orbit interaction between the ${ }^{3} \Pi$ state (with a ${ }^{4} \Pi$ core) and the ${ }^{1} \Sigma^{+}$state (with $\mathrm{A}^{2} \Sigma^{+}$core) is four times larger than in $\mathrm{HCl}$ (as the splitting between the cores $\mathrm{X}^{2} \Pi$ ) and is equal to $2080 \mathrm{~cm}^{-1}$ for $\mathrm{HBr}$ as compared to $520 \mathrm{~cm}^{-1}$ for $\mathrm{HCl}$ (Lefebvre-Brion and Keller 1989). 


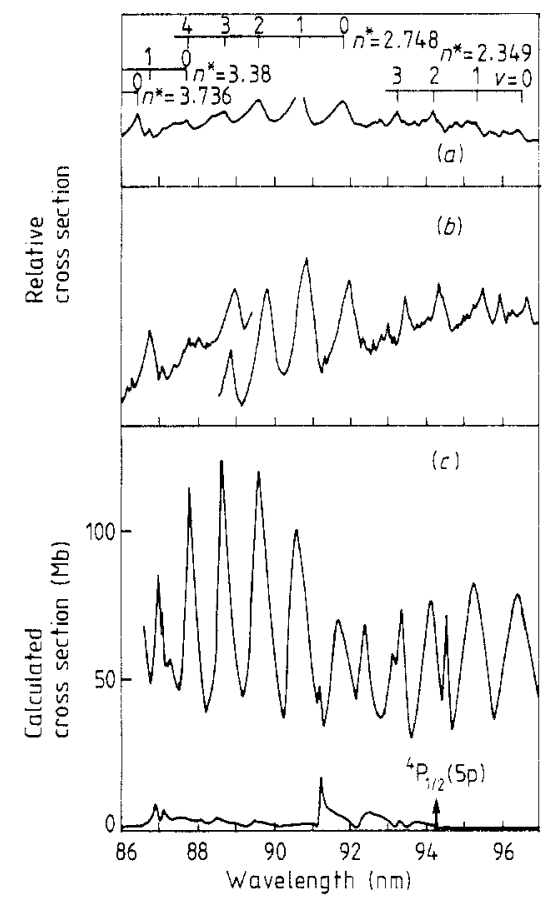

Figure 3. (a) Experimental photoionisation cross section with a resolution of $0.14 \bar{A}$ (Dehmer and Chupka 1978). (b) Experimental photoabsorption cross section with a resolution of $0.04 \AA$ (Terwilliger and Smith 1974). (c) Calculated photoionisation (top) and photodissociation cross sections (bottom).

The photoelectron angular distribution parameter $\beta$ and the spin-polarisation parameter $A$ were calculated from the transition moments.

\section{Theoretical results and comparison with experiment}

Figure 3 reports both the calculated photoionisation and photodissociation cross sections. The photoionisation cross section compares rather well with the experimental cross sections for photoabsorption (Terwilliger and Smith 1974, 1975) and photoionisation (Dehmer and Chupka 1978), after the quantum defects of the ${ }^{1} \Pi$ states have been slightly modified to fit the peak positions in the photoionisation cross section (see table 1 ). It is seen that the most intense autoionisation peaks have the ' $\Pi$ symmetry and are $5 \mathrm{p} \pi$ (progression II of Terwilliger and Smith 1975) and $4 \mathrm{~d} \pi$ (progression I). The calculated photodissociation cross section is due mainly to the ' $\Sigma^{+}$states, here the $4 \mathrm{~d} \sigma$ state. It is predicted to culminate at about $10 \mathrm{Mb}$, i.e. an order of magnitude larger than in $\mathrm{HCl}$, as predicted (Lefebvre-Brion and Keller 1989). These results depend strongly on the quantum defects of the ${ }^{1} \Sigma^{+}$states, which have been taken from the calculation, and on the shape of the predissociating states, and comparison with experimental results would be very interesting. From the comparison between experiment and calculation of the relative intensity of the peaks of the $5 \mathrm{~d} \pi$ progression, it is possible that the $5 \mathrm{~d} \pi$ state be strongly predissociated. It would be necessary in our model to include the dissociative state going to the $\mathrm{H}+\mathrm{Br}^{*}\left({ }^{4} \mathrm{D}_{1 / 2}, 5 \mathrm{p}\right)$ limit. The 
photoelectron angular distribution parameter $\beta$ has been calculated with and without including predissociation (see figures $4(a)$ and $(b)$ ). As expected, the influence of predissociation of the $4 \mathrm{~d} \sigma$ state can be seen in the $94-91 \mathrm{~nm}$ wavelength region as a small variation. Note that the calculations have been made with the transition moments calculated for $\varepsilon=0.005 \mathrm{au}$. If the calculations are made with the transition moments calculated for the actual value of $\varepsilon$, we estimate that $\sigma$ would be practically unchanged and $\beta$ would increase by about $20 \%$. The strong variation of $\beta$ observed in this energy domain (Raseev et al 1987) is due mainly to the energy dependence of the Coulomb phaseshift. For comparison with the structures predicted in figure 4 , it would also be interesting to perform measurements of the parameter $\beta$. However, the influence of the predissociation on $\beta$ as calculated in the energy region considered is too small to be detected experimentally.

Calculations have also been performed including only the $v=0$ level of $\mathrm{X}^{2} \Pi$. The electrostatic interactions between the $\mathrm{A}^{2} \Sigma^{+}$and the $v=0 \mathrm{X}^{2} \Pi$ channel have been weighted by the vibrational overlap given in the fourth column of table 2 . With these results, the $A$ (or $\bar{P}$ ) spin-polarisation parameter has been calculated (see figure 5 ) for $v=0$ of $\mathrm{X}^{2} \Pi_{1 / 2}$ in order to compare with the experimental data. Note that its value is given for the $X^{2} \Pi_{1 / 2}$ substate by the formula (Raseev et al 1987, equation (31))

$$
\bar{P}(1 / 2)=A(1 / 2)=\left(\sigma_{\Pi}^{\sigma}-\sigma_{\Pi}^{\delta}\right) /\left(2 \sigma_{\Pi}\right) .
$$

Thus the contribution to $A$ (or $\bar{P}$ ) of the autoionisation peaks in the numerator of equation (1) comes only from the ${ }^{1} \Pi$ states, which are autoionised either in the $\left(\mathrm{X}^{2} \Pi\right)$

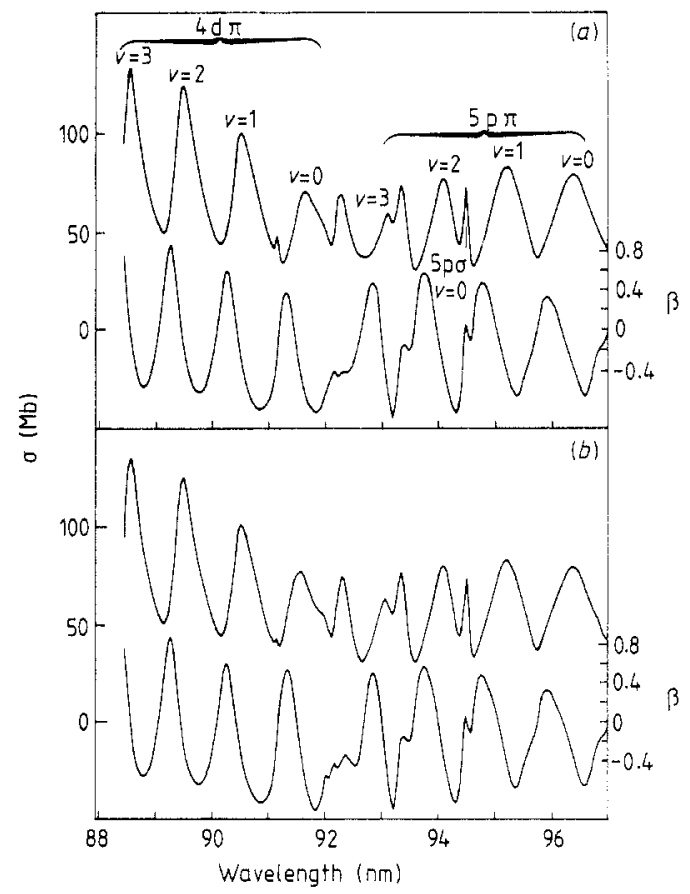

Figure 4. (a) Calculated photoionisation cross section, with predissociation (top) and calculated photoelectron angular distribution parameter $\beta$, with predissociation (bottom). (b) Calculated photoionisation cross section, without predissociation (top) and calculated photoelectron angular distribution parameter $\beta$, without predissociation (bottom). 


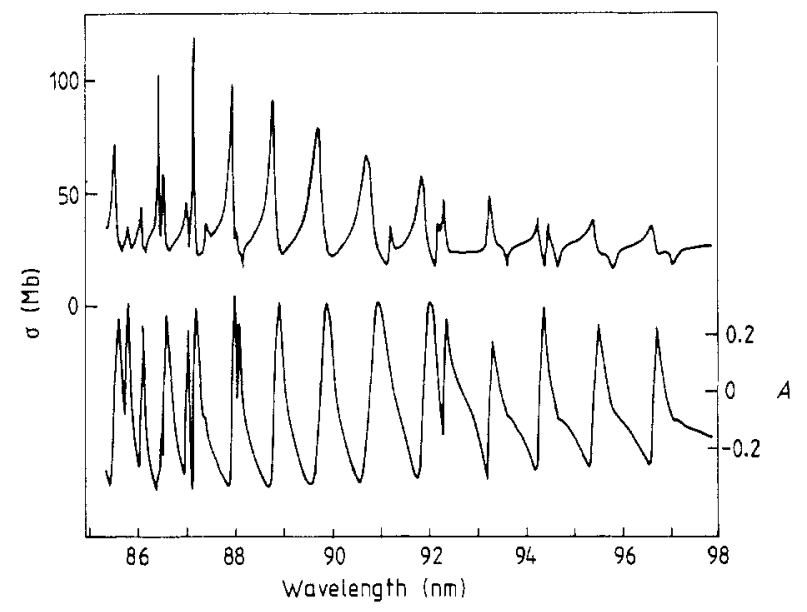

Figure 5. Calculated photoionisation cross section, without predissociation, for $v=0$ of $\mathrm{X}^{2} \Pi_{1 / 2}$ (top) and calculated spin-polarisation parameter $A$ (bottom).

$\varepsilon \sigma$ or $\left(\mathrm{X}^{2} \Pi\right) \varepsilon \delta$ continua of ${ }^{1} \Pi$ total symmetry (see table 1 ). Since the experimental results of $A$ in this energy region show a deviation from the calculated smooth curve without autoionisation, this is indirect proof that the autoionised peaks are mainly of ${ }^{1} \Pi$ symmetry.

In figure 6, the experimental results (see figure 1) are compared to the calculated results convoluted to a resolution of $500 \mathrm{~cm}^{-1}$. Since in general relation (1) was verified, we show in figure $6 A(1 / 2)$ and $-A(3 / 2)$. Qualitative agreement is found between theory and experiment. In particular, owing to the inclusion of electronic autoionisation, the theoretical results correctly predict a deviation of $A$ from the calculated value of about -0.2 for the open continuum (Raseev et al 1987). This is also observed in the experiment.

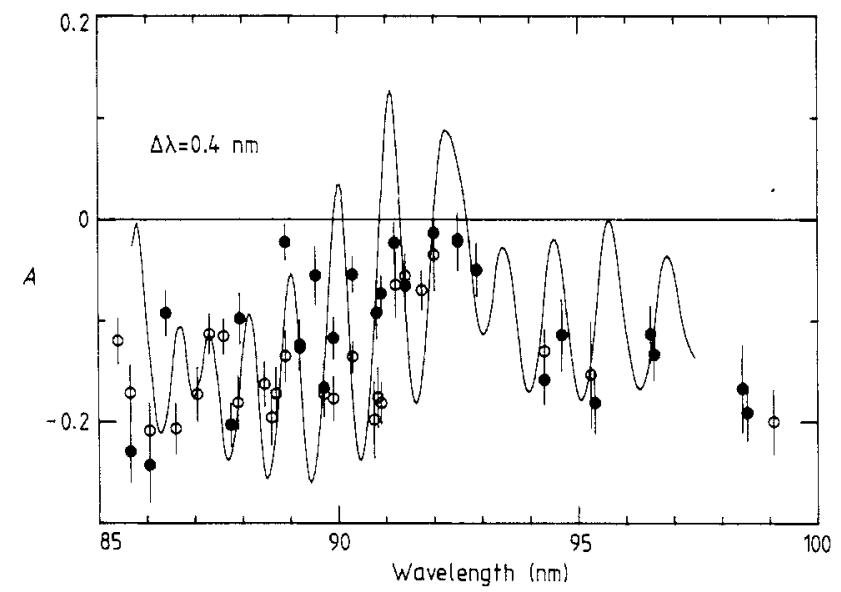

Figure 6. Calculated spin-polarisation parameter $A$ convoluted to the resolution of $500 \mathrm{~cm}^{-1}$ in comparison with the experimental data. The data points shown are $A(1 / 2)$ (full circles) and $-A(3 / 2)$ (open circles). 
The experimental data at the present stage are not sufficiently dense to outline completely the wavelength dependence of $A$ in full detail; however, a strong variation in the photon energy range examined is clearly indicated. A comparison of the individual data points with the theoretical prediction can be made: most of them lie on or close to the theoretical curve, although some deviations do exist. The convolution of the calculated curve for $A$ to the experimental resolution caused the amplitude of the oscillations to decrease (as can be seen from a comparison of figure 6 with figure 5 , where $A$ is shown without convolution). The oscillatory shape seems to be more distinct in the calculation than experimentally observed. However, in the photoionisation cross section (see figure 3 ), the ratio of the oscillation amplitude to the background appears to be too large in the calculation when compared to the experimental results. Since $A$ is a ratio of partial cross sections, this could in part explain why the experimentally observed oscillations for $A$ are less pronounced than calculated.

The cause of the oscillatory shape for $A$ is illustrated in figure 7 where the partial contributions $\sigma_{\Pi}^{\sigma}$ and $\sigma_{\Pi}^{\delta}$ are plotted as a function of energy. They oscillate with different phases and consequently it can be understood why $A$ is also an oscillating function of energy. From figure 7 it can also be comprehended why the theory predicts the structures for $A$ to be slightly shifted in wavelength compared to the partial cross section: to calculate the parameter $A(1 / 2)$, the partial contribution $\sigma_{\Pi}^{\delta}$ has to be subtracted from $\sigma_{\Pi}^{\sigma}$ according to equation (2), whereas in order to calculate the cross section the partial contributions have to be added.

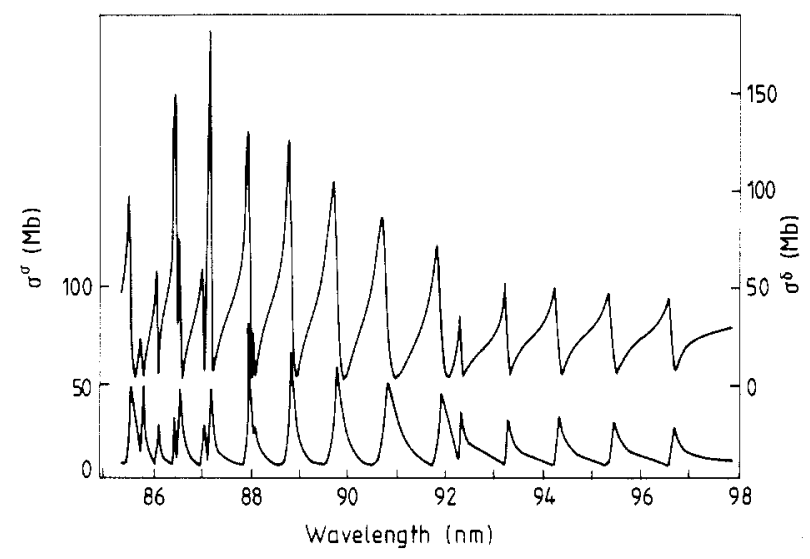

Figure 7. Values of $\sigma_{\Pi}^{\prime r}$ (bottom) and $\sigma_{\Pi}^{\delta}$ (top) as a function of energy.

\section{Conclusions}

In conclusion, the results illustrate the necessity to include the influence of autoionisation and predissociation in the calculation and interpretation of the experimental findings. Further, experiments would be very useful in order to verify the theoretical predictions both for the dissociation cross sections and for the $\beta$ parameter. For the spin-polarisation parameter $A$, more extensive experiments are planned. Measurements for $A$ were also made for the HI molecule (Böwering et al 1989). Unfortunately, for this molecule, the width of the resonances is of the order of magnitude of the vibrational 
interval and it would be necessary to modify the theory used here by the introduction of coupled equations.

\section{Acknowledgments}

One of the authors (HLB) thanks F Keller for useful discussions, G Raseev and H Le Rouzo for making available their program for the calculation of the ab initio parameters. The other authors would like to thank the BESSY staff, in particular F Schäfers, for cooperation and acknowledge support by BMFT (05 331 and $437 \mathrm{AXI}$ ). We thank $\mathrm{S}$ Peyerimhoff and A Banichevich for sending the calculated potential curves of $\mathrm{HBr}^{+}$ prior to publication. This joint research was supported by the European Commission.

\section{References}

Banichevich A, van Hemert M C and Peyerimhoff S 1989 to be submitted

Böwering N, Huth T, Mank A, Müller M, Schönhense G, Wallenstein R and Heinzmann U 1988 ElectronMolecule Scattering and Photoionisation ed P G Burke and J B West (New York: Plenum) pp 139-46

Böwering N, Müller M, Salzmann M, Klausing H W and Heinzmann U 1989 to be published

Cherepkov N A 1981 J. Phys. B: At. Mol. Phys. $142165-77$

Dehmer P M and Chupka W A 1978 Argonne National Laboratory Report ANL-78-65, part I, pp 13-18

Frohlich H, Glass-Maujean M and Guyon P M 1989 J. Chem. Phys, to be submitted

Giusti A 1980 J. Phys. B: At. Mol. Phys. $133867-94$

Giusti-Suzor A and Jungen Ch 1984 J. Chem. Phys. 80 986-1000

Heckenkamp Ch, Schäfers F, Schönhense G and Heinzmann U 1986 Z. Phys. D $2257-74$

Heinzmann U, Osterheld B, Schäfers F and Schönhense G 1981 J. Phys. B: At. Mol. Phy's. 14 L79-84

Heinzmann U, Schäfers F and Hess B A 1980 Chem. Phys. Lett. 69 284-9

Huber K P and Herzberg G 1979 Molecular Spectra and Molecular Structure vol IV Constants of Diatomic Molecules (New York: Van Nostrand-Reinhold)

Jost K 1979 J. Phys. E: Sci. Instrum. 12 1006-12

Lefebvre-Brion H, Dehmer P M and Chupka W A 1986 J. Chem. Phys. 85 45-50

1988 J. Chem. Phys. $88811-17$

Lefebvre-Brion $\mathrm{H}$ and Keller F 1989 J. Chem. Phys. $907176-83$

Raseev G, Keller F and Lefebvre-Brion H 1987 Phys. Rev. A 36 4756-74

Schäfers F, Peatman W, Eyers A, Heckenkamp Ch, Schönhense G and Heinzmann U 1986 Rev. Sci. Instrum. 57 1032-41

Terwilliger D T and Smith A L 1974 J. Mol. Spectrosc. 50 30-7 1975 J. Chem. Phys. 63 1008-20

White M G, Leroi G E, Ho M H and Poliakoff E D 1987 J. Chem. Phys. 87 6553-8 